\title{
Evaluación entre dos tecnologías usando Técnicas de Aglutinación en Columna (TAC) para la titulación e identificación de aloanticuerpos anti-eritrocitarios (columnas de micro-esferas de cristal y columnas de gel).
}

\section{Evaluation between two technologies using Column Agglutination Techniques \\ (TAC) for the tittration and identification of anti-erythrocytic alloantibodies (columns of glass microspheres and gel columns).}

*Correspondencia:

jenniffer.padilla@gmx.es

Teléfono [593] 032498288

Conflicto de intereses: Los autores declaran no tener conflictos de intereses.

Fondos: Ver la página 47

Recibido: 1 Enero 2018

Aceptado: 11 Abril 2018

Publicado: 30 Abril 2018

\section{Membrete bibliográfico:}

Padilla J, Maldonado B. Evaluación entre dos tecnologías usando Técnicas de Aglutinación en Columna (TAC) para la titulación e identificación de aloanticuerpos anti-eritrocitarios (columnas de micro-esferas de cristal y columnas de gel). Rev. Oncol. Ecu 2018;28(1):41-49.

DOI: https://doi.org/10.33821/251

Copyright Padilla, et al. Este artículo es distribuido bajo los términos de Creative Commons Attribution License, el cual permite el uso y redistribución citando la fuente $y$ al autor original.
Jenniffer Padilla Parra 1* iD, Bella Maldonado1.

1. Instituto Oncológico Nacional "Dr. Juan Tanca Marengo", Solca-Guayaquil, Laboratorio Clínico, Servicio de Medicina Transfusional y Banco de Sangre.

\section{Resumen}

Introducción: La presencia de anticuerpos anti eritrocitarios disminuye la sobrevida de los eritrocitos mediante la presencia de enfermedad hemolítica post transfusional en los pacientes oncológicos. La selección de la tecnología más conveniente para la determinación de los anticuerpos antieritrocitarios está directamente relacionada con la prevención de las reacciones transfusionales hemolíticas que son las responsables de la morbilidad y mortalidad relacionadas con la transfusión. El objetivo del presente estudio fue valorar la asociación estadística entre la técnica tradicional del gel versus la técnica de microesferas de cristal.

Métodos: El estudio fue realizado en el laboratorio Clínico del Instituto Oncológico Nacional Dr. Juan Tanca Marengo, Solca-Guayaquil el período de estudio enero 2017 a marzo del 2017. Se realizaron técnicas de titulación a partir de muestras de sangre periférica con aloanticuerpos anti-eritrocitarios de especificidad única que habían sido detectados durante la realización de las pruebas pretransfusionales. Las muestras fueron procesadas con la tecnología conocida usando la Técnica de Aglutinación en Columnas, columnas con gel. Posterior a realizar las pruebas pretransfusionales en los casos que presentaran positiva la prueba de escrutinio de anticuerpos o test de Coombs indirecto, inmediatamente después se llevaba a cabo la identificación del anticuerpo usando la misma técnica. 
Simultáneamente se realizaba las mismas pruebas usando la misma muestra con la otra tecnología de microesferas cristalizadas. Las reacciones de aglutinación se clasificaron como fuertemente positivas $(4+$ y $3+)$, moderadamente positivas $(2+$ y $1+)$ y positivo débil $(w+)$. El análisis estadístico se utilizó correlación de Spearman.

Resultados: En el período de estudio se efectuaron detección e identificación en 9 muestras. Seis muestras mostraron títulos idénticos, para ambas tecnologías, en 2 muestras se mostró títulos más altos con la tecnología en gel, y en 1 muestra se observó títulos más altos con la tecnología en micro esferas de cristal. La asociación Mediante Rho de Spearman entre las dos pruebas fue de $R=0.84$; $\mathrm{r} 2=0.72, \mathrm{P}=0.005$.

Conclusión: Existen una buena asociación entre las técnicas de gel y microesferas de cristal para la identificación de anticuerpos antieritrocitarios.

Palabras Claves: HEMÓLISIS, TÉCNICA DE PLACA HEMOLÍTICA, ANTICUERPOS.

DOI: $10.33821 / 251$

\section{Abstract}

Introduction: The presence of anti-erythrocyte antibodies reduces the survival of erythrocytes by the presence of post-transfusion hemolytic disease in cancer patients. The selection of the most convenient technology for the determination of antierithrocytic antibodies is directly related to the prevention of hemolytic transfusion reactions that are responsible for the morbidity and mortality related to transfusion. The aim of the present study was to evaluate the statistical association between the traditional gel technique versus the crystal microsphere technique.

Methods: The study was conducted in the Clinical Laboratory of the Dr. Juan Tanca Marengo National Oncological Institute, Solca-Guayaquil during the study period January 2017 to March 2017. Titration techniques were performed from peripheral blood samples with anti-alloantibodies-erythrocytes of unique specificity that had been detected during the pre-transfusion tests. The samples were processed with the known technology using the Agglutination Technique in Columns, columns with gel. After carrying out the pretransfusion tests in cases that presented a positive antibody test or indirect Coombs test, the antibody was immediately identified using the same technique. Simultaneously, the same tests were performed using the same sample with the other crystallized microsphere technology. The agglutination reactions were classified as strongly positive (4+ and $3+)$, moderately positive $(2+$ and $1+)$ and weak positive $(w+)$. The statistical analysis was used Spearman's correlation.

Results: In the study period, detection and identification were made in 9 samples. Six samples showed identical titers, for both technologies, 2 samples showed higher titers with gel technology, and in 1 sample higher titers were observed with the technology in crystal micro spheres. The association by Rho of Spearman between the two tests was $R=0.84 ; r 2=0.72, P=0.005$.

Conclusion: There is a good association between gel techniques and crystal microspheres for the identification of antierithrocytic antibodies.

Keywords: HEMOLYSIS, HEMOLYTIC PLATE TECHNIQUE, ANTIBODIES.

DOI: $10.33821 / 251$ 


\section{Introducción}

La presencia de anticuerpos anti eritrocitarios disminuyen la sobrevida de los eritrocitos mediante la presencia de enfermedad hemolítica post transfusional en los pacientes oncológicos y también se presenta en la enfermedad hemolítica del feto y del recién nacido [1-3]. La selección de la tecnología más conveniente para la determinación de los anticuerpos antieritrocitarios está directamente relacionada con la prevención de las reacciones transfusionales hemolíticas que son las responsables de la morbilidad y mortalidad relacionadas con la transfusión [4-5] y los anticuerpos no-ABO están frecuentemente implicados en reacciones hemolíticas transfusionales según informes de la FDA [6]. La titulación es un método semicuantitivo para estimar la fuerza y la concentración de los anticuerpos presentes en una muestra. Sus aplicaciones incluyen la determinación de la reactividad de los aloanticuerpos en el suero de pacientes poli transfundidos y obstétricas con historia de aloinmunización a antígenos eritrocitarios.

Los títulos consistentes y precisos son importantes debido a que el resultado puede inducir a la realización de la evaluación de la anemia en estos pacientes por métodos no invasivos como es el frotis de sangre periférico, la bioquímica sanguínea y recuento de reticulocitos. Recientemente las técnicas de aglutinación en columna en gel han dado lugar a la aparición de publicaciones en las que muestran datos y resultados confiables [7-12]. El alcance de este estudio es determinar hasta dónde es comparable la tecnología en gel con los resultados de la tecnología en micro-esferas de cristal en la titulación e identificación de aloanticuerpos pertenecientes a los diferentes sistemas que forman los múltiples grupos sanguíneos, que no sean del ABO y RhD analizados y estudiados siempre.

El método usado en la Sociedad de Lucha Contra el Cáncer (SOLCA) - Guayaquil para la identificación de anticuerpos anti- eritrocitarios también llamado anticuerpos irregulares es la Técnica de Aglutinación en Columna (TAC) manual, en gel. A propósito de este estudio se evaluaron otra tecnología con la misma técnica TAC con micro-esferas de cristal que prometen ser sensible, específico, ahorro de tiempo del operario y sobre todo de menor costo.

El objeto de este estudio es comparar y evaluar ambas tecnologías y así saber si la nueva tecnología es igual de sensible y específica que la ya conocida por la institución durante 10 años en la investigación de anticuerpos irregulares para las pruebas pre transfusionales.

\section{Materiales y Métodos}

El estudio fue realizado en el laboratorio Clínico del Instituto Oncológico Nacional Dr. Juan Tanca Marengo, Solca-Guayaquil el período de estudio enero 2017 a marzo del 2017. Se realizaron técnicas de titulación a partir de muestras de sangre periférica con aloanticuerpos anti-eritrocitarios de especificidad única que habían sido detectados durante la realización de las pruebas pre-transfusionales. Las muestras proceden de los pacientes oncológicos que se encuentran recibiendo tratamiento quimioterapia y hemoterapia en SOLCA Guayaquil. Estas muestras fueron tomadas en la sala de toma de 
muestras en el Banco de Sangre del Hospital. Luego de la centrifugación, las muestras fueron procesadas con la tecnología conocida usando la Técnica de Aglutinación en Columnas, columnas con gel. Posterior a realizar las pruebas pretransfusionales en los casos que presentaran positiva la prueba de escrutinio de anticuerpos o test de Coombs indirecto, inmediatamente después se llevaba a cabo la identificación del anticuerpo usando la misma técnica. Simultáneamente se realizaba las mismas pruebas usando la misma muestra con la otra tecnología y de esa manera se comparaba los resultados [10, 11]. La tecnología conocida de Bio-Rad (TAC con gel) recomienda para la identificación de anticuerpos irregulares se desarrolle utilizando las tarjetas (Coombs-IgG + complemento C3) frente a un panel de células comerciales ID- DiaPanel (11 viales) en una concentración de $0.8 \%$ según las recomendaciones del fabricante. Las tarjetas fueron centrifugadas a $1030 \mathrm{rpm}$ durante 10 minutos previo a la realización de la prueba, posterior se identifica la tarjeta con los datos del paciente y con la nomenclatura que le corresponde a cada pocillo, se procede a quitar el sello de seguridad de las tarjetas y se empieza a colocar las células conocidas de los viales antes mencionados (11 viales) en los pocillos correspondientes. Posterior se coloca el plasma del paciente luego se realiza la incubación de 15 minutos a $37^{\circ} \mathrm{C}$ y se empieza a colocar en la ID-Centrifugue 12SII para ser centrifugadas a $1030 \mathrm{rpm}$ durante 10 minutos.

Las reacciones de aglutinación se clasificaron como fuertemente positivas (4+ y $3+$ ), moderadamente positivas $(2+$ y $1+)$ y positivo débil $(w+) .13$ Las células utilizadas fueron ID- DiaCell I, II, III para detección de aloanticuerpos y las células ID- DiaPanel (11 viales) para identificación de los anticuerpos.

La tecnología nueva de Ortho clinical diagnostig (TAC con micro-esferas de cristal) recomienda para la detección e identificación de anticuerpos irregulares se desarrolle utilizando cassettes (Coombs-IgG + complemento C3) frente a un panel de células comerciales Selectogen ( 3 viales) en una concentración de $0,8 \%$ según las recomendaciones del fabricante y usamos ID- DiaPanel (11 viales) de la casa comercial BioRad. Los cassettes se identificaron con los datos del paciente y con la nomenclatura que le corresponde a cada pocillo, se procede a quitar el sello de seguridad de los cassettes y se empieza a colocar las células conocidas de los viales antes mencionados, en el pocillo correspondiente. Posterior se coloca el plasma del paciente luego se realiza la incubación por 10 minutos a $37^{\circ} \mathrm{C}$ y se empieza a colocar en la centrifuga durante $5 \mathrm{~min}$. Se utiliza la asociación estadística con el coeficiente de Ro de Spearman, la asociación significativa se determina con un valor $P$ menor a 0.05 . El paquete estadístico utilizado fue SPSS 22.0 para PC.

\section{Resultados}

En el período de estudio se efectuaron detección e identificación en 9 muestras con las dos técnicas gel y Micro esferas de cristal (Tabla 1). Seis muestras mostraron títulos idénticos, para ambas tecnologías, en 2 muestras se mostró títulos más altos con la tecnología en gel, y en 1 muestra se observó títulos más altos con la tecnología en micro esferas de 
cristal. Los resultados de la identificación fueron idénticos (Tabla 2). La asociación Mediante Rho de Spearman entre las dos pruebas fue de $R=0.84 ; r^{2}=0.72, P=0.005$ (Tabla 3 y Figura 1).

Tabla 1. Especificidad de anticuerpos anti-eritrocitario.

\begin{tabular}{|l|c|c|l|l|}
\hline Especificidad & $\begin{array}{l}N^{\circ} \text { de Muestra } \\
\mathrm{n}=9\end{array}$ & $\begin{array}{l}\text { Idéntica } \\
\mathrm{n}=6\end{array}$ & $\begin{array}{l}\text { Mayor en } \\
\text { gel } \\
\mathrm{n}=2\end{array}$ & $\begin{array}{l}\text { Mayor en } \\
\text { micro-esferas } \\
\text { de cristal } \\
\mathrm{n}=1\end{array}$ \\
\hline Anti-C & 3 & 2 & - & 1 \\
\hline Anti-K & 3 & 2 & 1 & - \\
\hline Anti-Kp & 1 & - & 1 & - \\
\hline Anti-Lub & 2 & 2 & - & - \\
\hline
\end{tabular}

Tabla 2. Resultados de hemólisis comparativa entre las dos pruebas

\begin{tabular}{|l|c|c|}
\hline & Gel & $\begin{array}{c}\text { Micro esferas de } \\
\text { cristas }\end{array}$ \\
\hline Muestra 1 & 3,00 & 3,00 \\
\hline Muestra 2 & 4,00 & 4,00 \\
\hline Muestra 3 & 1,00 & 1,00 \\
\hline Muestra 4 & 2,00 & 2,00 \\
\hline Muestra 5 & 4,00 & 4,00 \\
\hline Muestra 6 & 4,00 & 4,00 \\
\hline Muestra 7 & 4,00 & 3,00 \\
\hline Muestra 8 & 3,00 & 2,00 \\
\hline Muestra 9 & 2,00 & 3,00 \\
\hline
\end{tabular}

Tabla 3. Asociación entre los resultados de hemólisis entre las técnicas en Gel y Microesferas de cristal.

\begin{tabular}{|c|c|c|}
\hline & & $\begin{array}{l}\text { Micro esferas } \\
\text { de cristal }\end{array}$ \\
\hline Rho de Spearman Gel & $\begin{array}{l}\text { Coeficiente de correlación } \\
\text { Sig. (bilateral) } \\
\mathrm{N}\end{array}$ & $\begin{array}{c}0.840^{* *} \\
0.005 \\
9\end{array}$ \\
\hline
\end{tabular}


Figura 1. Dispersograma entre las dos pruebas para la identificación de hemólisis.

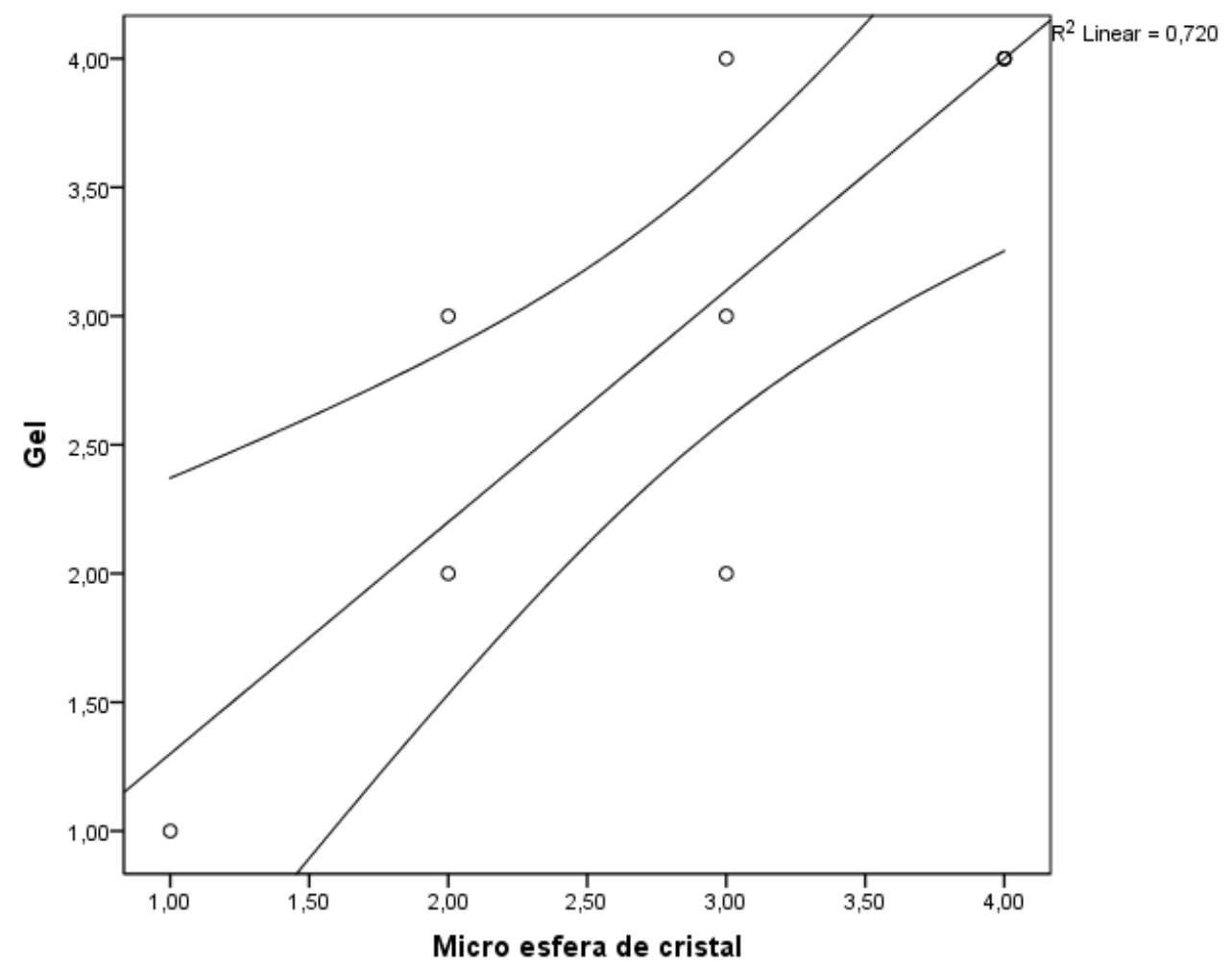

\section{Discusión}

La aloinmunización tiene un impacto negativo y significativo en los recursos del laboratorio de inmunohematología e institucional asociado con la necesidad de incrementar las pruebas para la identificación de unidades compatibles para transfusión y en el manejo obstétrico, con la evaluación y manejos de las reacciones transfusionales. Está clara la importancia de este tipo de estudios en la población a transfundir y se pone en evidencia la gran utilidad de definir la mejor batería de técnicas según las posibilidades del centro $[11,12]$. A pesar de que más de 50 especificidades diferentes han sido implicadas en la patogénesis de la enfermedad hemolítica del feto y el recién nacido, aquellos dirigidos contra antígenos de los Sistemas Rh y Kell son los más comúnmente asociados con una mayor probabilidad de anemia fetal severa, aunque en el presente estudio no se ha evaluado estos casos clínicos. En todos estos casos es notable la importancia de realizar estudios de titulación para estimar su fuerza y concentración $[7,10]$. Aunque el título no parece ser suficiente para predecir la severidad de la hemólisis en los pacientes oncológicos o neonatos, es conveniente conocer el llamado "título crítico" comúnmente solicitado para tomar la decisión de monitorear por métodos no séricos. Por otro lado, se han informado reacciones hemolíticas postransfusionales tardías causadas por aloanticuerpos anti-D, -C, -K, -Jka y -E en combinación con -Fya así como de anti-K y anti$\mathrm{Di}^{\mathrm{a}}[11-14]$. 
La técnica recomendada por la AABB para este propósito es el método convencional de titulación en tubo a pesar de los problemas para estandarizar esta técnica que incluye las diferentes condiciones interlaboratoriales de equipo, preparación de las muestras, antisueros y dependencia del operador [15].

Actualmente se ha determinado en varios estudios que las diferencias entre los resultados de ambas tecnologías en microcolumna es menor de lo que se esperaría. Esta diferencia no es considerada como significativa $[16,17]$ y la variación entre las dos tecnologías no excede lo que es considerado como aceptable. Las muestras utilizadas en este estudio provienen de pacientes aloinmunizados y no hay diferencia en la dilución de los glóbulos rojos utilizados para ambas técnicas, las lecturas fueron realizadas por la misma persona. Sin embargo, se puede aportar que la técnica de aglutinación en microcolumnas con microesferas de cristal puede ser evaluada como una opción para la titulación de anticuerpos.

\section{Conclusiones}

Existe una buena asociación entre las técnicas de gel y microesferas de cristal para la identificación de anticuerpos antieritrocitarios.

\section{Agradecimientos}

Reconocemos a las personas que participaron indirectamente en el estudio tales como el personal técnico, del Instituto Oncológico Nacional "Dr. Juan Tanca Marengo", SolcaGuayaquil, Laboratorio Clínico, Servicio de Medicina Transfusional y Banco de Sangre.

Nota del Editor

La Revista Oncología Ecu permanece neutral con respecto a los reclamos jurisdiccionales en mapas publicados y afiliaciones institucionales.

\section{Información adicional}

\section{Abreviaturas}

AABB: Asociación Americana de Bancos de Sangre.

TAC: Técnica de Aglutinación en Columna.

\section{Archivos Adicionales}

Ninguno declarado por los autores.

Fondos

Los fondos de la investigación fueron propios de los autores del presente artículo. 


\section{Disponibilidad de datos y materiales}

Existe la disponibilidad de datos bajo solicitud al autor de correspondencia. No se reportan otros materiales.

\section{Contribuciones de los autores}

JP, BM realizaron la idea de investigación, revisión bibliográfica. JP, recolección de datos, escritura del artículo. BM realizó el análisis crítico del artículo. Todos los autores leyeron y aprobaron la versión final del artículo.

Aprobación de ética y consentimiento para participar

No aplica a este estudio.

\section{Consentimiento para publicación}

No aplica.

\section{Información de los autores}

Jenniffer Padilla Parra, Hematóloga Clínica del Banco de Sangre, Instituto Oncológico Nacional "Dr. Juan Tanca Marengo", Solca-Guayaquil., Laboratorio Clínico, Servicio de Medicina Transfusional y Banco de Sangre. (iD https://orcid.org/0000-0002-6813-8183

Bella Maldonado, Hematóloga Clínica y Trasplante de Medula Ósea - Jefe del Servicio. , Instituto Oncológico Nacional "Dr. Juan Tanca Marengo", Solca-Guayaquil., Laboratorio Clínico, Servicio de Medicina Transfusional y Banco de Sangre.

\section{Revisiones por pares}

Acceda a la revisión de pares académicos en el siguiente enlace: https://publons.com/review/3820698

\section{Referencias}

Abreviaturas en la referencias DOI: Digital Object Identifier PMID: PubMed Identifier SU: Short URL
1. Downes K, Shulman I. Pretransfusion testing. En: Roback J, Combs M, Grossman B, Hillyer C, eds. Technical Manual 16th ed. Arlington, VA: America Association of Blood Banks, 2008: 437-63 
2. Haywood J, Moulds M, Bryant B. Determination of optimal method for antibody identification in a reference laboratory. Inmunohematology 2011; 27(4): 146-150. PMID: 22646070

3. Winters J, Richa E, Bryant S, Tauscher C, Bendix B, Stubbs J. Polyethylene glycol antiglobulin tube versus gel microcolumn: influence on the incidence of delayed hemolytic transfusion reactions and delayed serologic transfusion reactions. Transfusion 2010;50(7):1444-52. DOI: $10.1111 / \mathrm{j} .1537-$ 2995.2010.02609.x.

4. Linares J. Inmunohematología y transfusión: principios y procedimientos. Cromotip; 1986. 146-150 p. ISBN: 978-980-265-332-4

5. Linden J, Wagner K, Voytovich A, Sheehan J. Transfusion errors in New York State: an analysis of 10 years' experience. Transfusion. 2000;40(10):1207-13. DOI: 10.1046/j.1537-2995.2000.40101207.x

6. Powers A, Chandrashekar S, Mohammed M, Uhl L. Identification and evaluation of false-negative antibody screens: FALSE-NEGATIVE ANTIBODY SCREENS. Transfusion. 2009;50(3):617-21. DOI: 10.1111/j.1537-2995.2009.02464.X

7. Finck R, Lui-Deguzman C, Teng S-M, Davis R, Yuan S. Comparison of a gel microcolumn assay with the conventional tube test for red blood cell alloantibody titration. Transfusion. 2013;53(4):811-5. DOI: 10.1111/j.1537-2995.2012.03793.x

8. Kumlien G, Wilpert J, Säfwenberg J, Tydén G. Comparing the tube and gel techniques for ABO antibody titration, as performed in three European Centers. Transplantation. 2007;84(Supplement):S17-9. DOI: 10.1097/01.tp.0000296019.85986.af

9. Cohney S, Hogan C, Haeusler M, Neander E, Fairweather H. Variability of anti-blood group titers according to methodology and clinical relevance in $\mathrm{ABO}$-incompatible renal transplantation. American journal of transplantation (conference paper). 2007: 157

10. Shirey RS, Cai W, Montgomery RA, Chhibber V, Ness PM, King KE. Streamlining ABO antibody titrations for monitoring ABO-incompatible kidney transplants. Transfusion. 2009;50(3):631- 34. DOI: 10.1111/j.1537-2995.2009.02478.x

11. Cerdas-Quesada C. Correlación entre dos técnicas para la detección e identificación de anticuerpos anti-eritrocitarios: polietilenglicol y columnas de gel. Revista Argentina de Transfusion. 2013; 39 (4): 261-264. SU: goo.gl/E88jyG

12. Cerdas-Quesada C. Correlación entre dos técnicas para detección e identificación de anticuerpos antieritrocitarios: polibrene y columnas de gel. Revista Argentina de Transfusión. 2013; 39 (1-2): 51-54. SU: goo.gl/bCeJej

13. Steiner E, Judd W, Combs M, Watkins K, Weissman P, Mann N, et al. Prenatal antibody titers by the gel test. Transfusion. 2001;41:31S-31S.

14. Marsh WL. Scoring of Hemagglutination Reactions. Transfusion. 1972;12(5):352-3. DOI: 10.1111/j.1537-2995.1972.tb04459.x

15. Steiner $E$, Judd $W$, Combs $M$, Watkins $K$, Weissman $P$, Mann $N$, et al. Prenatal antibody titers by the gel test. Transfusion. 2001;41:31S-31S. DOI: 10.1111/j.1365-3148.1995.tb00228.x

16. Thakur MK, Marwaha N, Kumar P, Saha SC, Thakral B, Sharma RR, et al. Comparison of gel test and conventional tube test for antibody detection and titration in D-negative pregnant women: study from a tertiary-care hospital in North India. Immunohematology. 2010;26(4):174-7. SU: goo.gl/eHLpSW

17. Arroyo A, Guerrero S, Rojo J. Desempeño del analizador automatizado para inmunohematología con tecnología de aglutinación en columna-fase sólida de esferas de cristal. Rev Mex Med Tran. 2011; 4(2): 127. SU: goo.gl/XtB9wf 